\title{
Optical resonances and field-enhancement in 3D metal nanorods
}

\author{
Thomas M. Søndergaard ${ }^{1}$
}

\author{
${ }^{1)}$ Department of materials and production, Aalborg University, Aalborg East, Denmark \\ *)E-mail ts@mp.aau.dk
}

In this paper the optics of single and coupled 3D metal nanorods in solution is considered theoretically using the Electric-Field Integral Equation (EFIE) [1]. The extinction, scattering, and absorption cross section spectra, and field enhancement distributions, will be presented at the conference for a range of single rod geometries, and for configurations of rods with e.g. varying lengths and separation.

An example of the extinction cross section spectra for single rods with a radius of $5 \mathrm{~nm}$ and different lengths $h$ is shown in Fig. 1(a). Here, the rods are illuminated with a plane wave polarized along the z-axis (along the rods) and propagating along the $\mathrm{x}$-axis. The resonance seen in the spectra clearly redshifts with the rod-length $h$, and it is thus possible to control the resonance via the geometry. An example of the enhancement of the magnitude of the electric field is shown in Fig. 1(b) for rods with the length $\mathrm{h}=30 \mathrm{~nm}$ at the resonance wavelength.

(a)

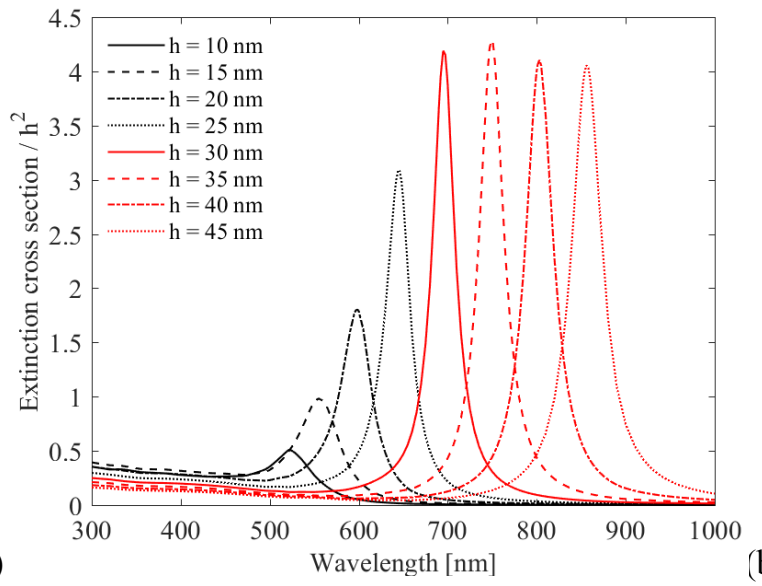

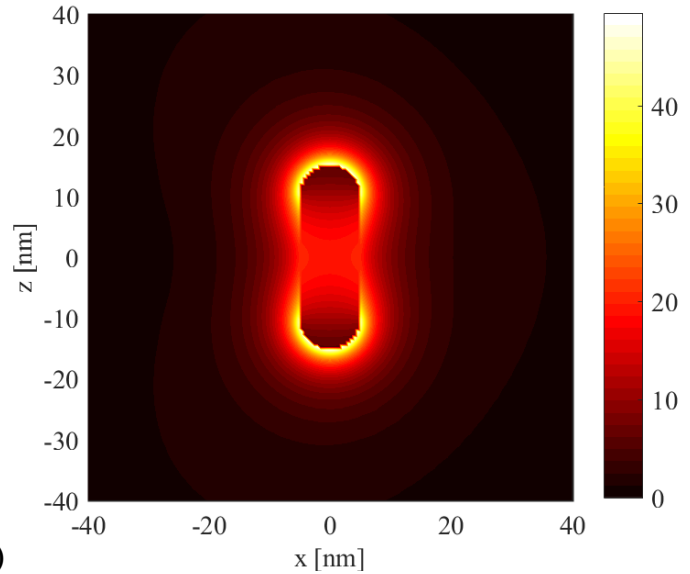

Figure 1. (a) Extinction cross section spectra of gold nanorods in water with radius $5 \mathrm{~nm}$ and different lengths $\mathrm{h}$. (b) Enhancement of the electric-field magnitude at resonance for $\mathrm{h}=30 \mathrm{~nm}$. The rods are illuminated with light polarized along the rod axis $(\mathrm{z})$.

At the conference similar calculations will also be presented for other rod geometries with e.g. flat ends instead of rounded ends and for configurations of two rods separated by a small or large gap. In the latter case the resonance wavelength will be shown to be highly sensitive to the gap size, and more so for rods with flattened ends. The field enhancement is on the other hand higher for rods with rounded ends. With two rods, each being similar to the one considered in Fig. 1(b), and with a 1-nm gap, the field enhancement at resonance is app. 400.

\section{References.}

[1] Thomas M. Søndergaard, "Green's Function Integral Equation Methods in Nano-Optics", CRC Press 2018. 